\title{
Visual outcome after posterior uveal melanoma episcleral brachytherapy including radiobiological doses
}

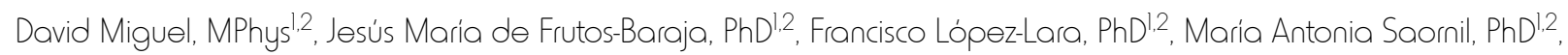 \\ Ciro García-Alvarez, PhD',2, Pilar Alonso, PhD',2, Patricia Diezhandino, PhD!.2 \\ IIntraocular Tumors Unit Valladolid University Hospital, Valladolid, ${ }^{2}$ University of Valladolid, Valladolid, Spain
}

\begin{abstract}
Purpose: To assess the long-term influence of radiobiological doses in the evolution of visual acuity (VA) in patients with uveal melanoma treated by episcleral brachytherapy.

Material and methods: Visual acuity was evaluated prospectively from a case series of 243 patients in 2016 treated with ${ }^{125} \mathrm{I}$. Data analysis was applied to trend VA outcome and find the accurate best-fit line. Biologically effective dose (BED) was included in survival analysis with the use of Kaplan-Meier and Cox regressions. Hazard ratio (HR) and confidence interval at $95 \%$ (CI) were determined. Variables statistically significant were analyzed and compared by log-rank tests.

Results: The median follow-up was 74.2 months (range, 3-223). Exponential regression shows a $25 \%$ reduction and $50 \%$ in visual acuity score (VAS) scale for 5 and 27.8 months, respectively. Cumulative probabilities of survival analysis were $57 \%, 42 \%, 27 \%$, and $23 \%$ at 3, 5, 10, and 15 years, respectively. Multivariable analysis found tumor height $(\mathrm{HR}=1.18,95 \% \mathrm{CI}: 1.07-1.29)$, applicator size $(\mathrm{HR}=1.22,95 \% \mathrm{CI}: 1.08-1.36)$, juxtapapillary localization $(\mathrm{HR}=1.70$, 95\% CI: 1.01-2.84), and dose to foveola (HR $=1.01,95 \% \mathrm{CI}: 1.00-1.01)$ significantly associated with VA loss. Log-rank tests were significant for all those variables. BED has a strong influence in univariate model, but not statistically significant in the multivariate one.

Conclusions: Visual acuity changes can be modeled by an exponential function for the first 5 years after treatment. No relation between VA loss and BED has been found; nevertheless, apical height, plaque size, juxtapapillary localization, and dose to fovea were found as statistical significant variables.

J Contemp Brachytherapy 2018; 10, 2: 123-131 DOI: https://doi.org/10.5114/jcb.2018.75597
\end{abstract}

Key words: brachytherapy, plaque brachytherapy, radiobiology, radiobiological doses, uveal melanoma.

\section{Purpose}

Uveal melanoma is the most frequent primary malignant intraocular tumor occurring in adults, with an annual age-adjusted incidence of 5.1 per million [1]. Approximately, $90 \%$ of all uveal melanomas' develops in the choroid, $7 \%$ in the ciliary body, and $3 \%$ in the iris [1]. In the past, uveal melanoma was treated with enucleation, but plaque brachytherapy played a major role in the management of posterior uveal melanoma since the Collaborative Ocular Melanoma Study (COMS) [2,3]. Following the COMS classification system, brachytherapy was indicated in the following three conditions: small melanomas with a documented tendency to grow or presented clear signs of activity, all medium-sized melanomas, and some large melanomas with a reasonable potential for visual conservation provided patient agreement [4].
The main clinical goals of brachytherapy are tumor control, eye preservation, maintenance of vision, and quality of life. Dose prescription target volume is defined by the apex of the tumor and a small retinal margin (typically $2 \mathrm{~mm}$ ) surrounding the tumor base [5] that accounts for microscopic extension of the tumor, as recommended by the American Brachytherapy Society (ABS) [6], and is usually defined as the planning target volume (PTV) [7].

The ABS recommended prescription dose is $85 \mathrm{~Gy}$ to the apex of the tumor using the COMS dosimetry assumptions and plaque construction techniques [6]. The $85 \mathrm{~Gy}$ isodose line should pass through the prescription point and encompass the cited PTV. The ABS suggest a dose rate of $0.60-1.05 \mathrm{~Gy} / \mathrm{h}$, delivering the total dose in 3 to 7 consecutive days. Following these guidelines, each institution should decide the best dose rate at the prescrip- 
tion point, such that the dose delivery is accomplished in a timely manner [8].

Although effective, this procedure may result in various ocular complications $[9,10]$, and it often leads to a significant loss of visual acuity (VA), since the treatment delivers a high radiation dose. There are several reason for it, but the main disorders associated are retinopathy, maculopathy, cataract, neovascular glaucoma, and nerve atrophy $[11,12,13,14,15,16,17]$. The severity of complications depends mainly on the amount of incidental irradiation to the respective tissues and radiosensitivity of ocular structures [18].

In this way, Dale [19] quantitatively evaluated the biologically effective dose (BED) of non-permanent brachytherapy implants as a function of several parameters: the implant duration, dose rate, radionuclide, and radiobiological parameters for the tumor and organs at risk (OAR) related with repair rates and radiosensibility. Gagne et al. applied this model and concluded that tumor and OAR dose-volume histograms were sensitive to implant duration, which may be manipulated to affect outcomes [20], and suggested that shorter implant durations may correlate with more favorable sequelae [21]. They also concluded that the radionuclide election could offer a substantial physical radiobiological dosimetric benefit [21]. However, BED has not yet been implemented in the analysis of clinical series of patients treated with brachytherapy plaques.

The aim of this study was to analyze the influence of BED on visual outcome for patients treated with ${ }^{125} \mathrm{I}$ brachytherapy at our center, and the correlation with recognized prognostic factors. A further aim was to identify risk factors for visual loss.

\section{Material and methods \\ Workflow}

The workflow in choroidal melanoma interventional radiotherapy was articulated in four main issues: 1 . Multidisciplinary tumor board: case presentation and choice of treatment; 2. Treatment planning: plan calculation and pre-plan approval; 3. Source preparation: applicator loading and sterilization; 4. Surgery: plaque implantation, treatment, and plaque removal [22].

\section{Patients and follow-up}

All patients were initially evaluated and diagnosed with uveal melanoma by an ophthalmologist with expertise in ocular oncology. Brachytherapy was performed according to the standard protocol of the American Brachytherapy Society (ABS) guidelines [6,8]. Ophthalmologist and oncologist contoured the target, and the plaque size was chosen to sufficiently encompass the basal margin.

Patients treated with ${ }^{125}$ I (ROPES [23] and COMS [24]) plaques for uveal melanoma were included in this study prospectively and consecutively from $1^{\text {st }}$ of January 1996 to $1^{\text {st }}$ of July 2016, at the intraocular tumor unit at Valladolid University Hospital (Spain). Patients treated with transpupillary thermotherapy prior to brachytherapy were excluded.
All patients signed an informed consent form after being appropriately informed about possible side effects. The tumors were localized by transillumination and indirect ophthalmoscopy. The applicators were sutured to the sclera and removed after expiration of recommended time. The prescribed dose at the tumor apex was $85 \mathrm{~Gy}$ in all cases.

Patient data included demographics (age and gender) and tumor characteristics (COMS size, apical height, longest basal dimension, laterality, length, latitude, location of anterior tumor border, location of posterior border, tumor shape, and juxtapapillary localization). In addition, all patients underwent a full ophthalmic examination, including Snellen visual acuity. Regular follow-up was scheduled at $1,3,6$, and 12 months, every 6 months from 1 to 5 years after therapy, and then annually thereafter, if local control had been achieved. In practice, the number of revisions for many patients may be more numerous during the first 5 years, mainly due to the special monitoring of some of them, and follow-up may vary due to hospital planning.

The study protocol was approved by the institutional research committee, in accordance with national data protection laws and with the ethical principles of the Helsinki Declaration.

\section{Visual acuity definition and percentage changes in visual acuity}

VA is defined as the reciprocal of the ratio between the letter size that can just be recognized by a patient, relative to the size just recognized by a standard eye. Preoperative and post-operative VA were registered in decimal scale $(\mathrm{V})$, which is a logarithmic. Complete loss of vision, in this manuscript, is defined as VAS $\leq 50(\mathrm{~V} \leq 0.1)$, what is the legal limit in Spain.

Linear scales are not meant for clinical records, but they are required for statistical manipulations [25]. They convert the progression of $\mathrm{V}$ values to a linear one, based on Weber-Fechner's law, which states that proportional stimulus increases lead to linear increases in perception [26]. One of the most used scales is the visual acuity score (VAS) is related to $\mathrm{V}$ as follows: $V A S=100+50 \log V$. This score is more intuitive, since higher values indicate better vision [25].

Percentage changes in VA were evaluated as $\Delta(t)=$ $100\left(V A S(t) / V A S_{0}\right)$, where $V A S_{0}$ and $V A S(t)$ are the VA in VAS scale initial and in the revision for each time $t$ of follow-up, respectively. Eyes enucleated after therapy were consider analogously to lost to follow-up, i.e., the penultimate VAS acquired before enucleation was maintained and subsequently, the patient was not included in the active cohort.

\section{Clinical target volume definition and dosimetry}

The radiation oncologist defined the clinical target volume (CTV) considering the tumor thickness read on B-scan sonography images and a safety margin extension of 1-2 mm in all directions. Planning target volume (PTV) can be added by the radiation oncologist in case of doubts in plaque localization or tumor delineation [27]. 
The three-dimensional reconstruction and dosimetry was performed by a computer system developed by Dr. Astrahan at the University of California (BEBIG Plaque Simulator, version 2.16) [28]. Seed Amersham model 6711 [29] and Bebig model I25.S16 [30] are used for ROPES and COMS plaques, respectively. Calculations are based on the reports of the American Association of Physicists in Medicine Radiation Therapy Committee Task Group No. $43[31,32]$ for iodine plaques. Plaque heterogeneity correction functions were incorporated in the treatment planning. Dose collimation by the lip on the gold-alloy backing and global attenuation factor that considered the effect of the eye plaque seed carrier was also enabled.

Plaque arrangements can vary in source strengths and ring sizes [33]. An independent check of treatment time by redundant calculation was performed.

Before treatment, we determined the following information: treatment length, plaque size, number of seeds (in case of iodine plaque), total activity, and distribution of ${ }^{125} \mathrm{I}$ seeds required to provide the prescribed dose to the target volume. We also collected initial dose rates and doses to the prescription point, eye center (EC) - $12 \mathrm{~mm}$ from plaque center, sclera $-1 \mathrm{~mm}$ from plaque center, and critical structures within the eye: lens center of plaque to center of the lens, optic disc (OD) - center of plaque to center of $\mathrm{OD}$, and foveola - center of plaque to center of foveola.

\section{Biologically effective dose calculations}

The BED equation for temporary brachytherapy implants was established by Dale [19] and Dale \& Jones [34]. Furthermore, it was examined by Baltas et al. [35]. BED incorporates, among other factors, initial dose rate, radionuclide half-life, tissue type, and repopulation terms and take the following form:

$$
\begin{aligned}
\mathrm{BED}= & {\left[\frac{R_{0}\left(1-\mathrm{e}^{-\lambda T}\right)}{\lambda}\right]\left\{1+\frac{2 R_{0} \lambda}{(\mu-\lambda)(\alpha / \beta)\left(1-\mathrm{e}^{-\lambda T}\right)}\right.} \\
& {\left.\left[\frac{1}{2 \lambda}\left(1-\mathrm{e}^{-2 \lambda T}\right)-\frac{1}{\mu+\lambda}(1-\mathrm{e}-(\mu+\lambda) T)\right]\right\} }
\end{aligned}
$$

It is assumed that the repopulation rate remains constant and it is not needed for a late responding tissue [36]. Decay constant and all of the radiobiological values from Gagne et al. [20] were maintained.

\section{Statistics analysis}

Eligible patients for statistical analysis had VA better than $20 / 200$, i.e., V > 0.1 (> 50 in VAS scale). Relative visual loss for a time $t$ was calculated for each patient $(\mathrm{P})$ individually, $\Delta \_p(t)$; then, the mean variation for each quarter from treatment $(\mathrm{T})$ was estimated, $\Delta(T)$.

Scatter plot of $\Delta(T)$ against $\mathrm{T}$ was shown, and degree of statistical correlation between the variables in the visualization by fitting line plots was identify for the first 5 years of follow-up. In order to find the most accurate best-fit line, we were placing data on linear, quadratic hyperbolic, and exponential functions.

Secondly, the research focused on time to event, in which the patient lost vision (VAS $\leq 50$ ), therefore, the appropriate method of this particular study was survival analysis. Visual outcome over time were estimated with
95\% confidence intervals (CI) by Kaplan-Meier analysis [37], and VA preservation rates were reported at 3, 5, 10, and 15 years follow-up. The effect of individual clinical variables on the development of visual loss was analyzed by a series of univariate Cox proportional hazards regressions [38]. Hazard ratio (HR) and 95\% CI for variables that showed a degree of correlation $p<0.1$ were determined and included in a final multivariate model that fitted variables identified as significant predictors in the backward stepwise model.

Thirdly, Kaplan-Meier analysis and estimation of differences using the log-rank test [37] was performed for those quantitative and qualitative variables, which multivariate study of proportional hazards presented statistical significance. For quantitative variables, we shared the sample into its median value in order to ensure the same number of data in both branches. For the qualitative variables, a division was already made, and we analyzed each category.

All variables were analyzed as discrete except age, apical height, longest basal dimension, activity and treatment time, radiation doses, and BED, which were analyzed as continuous variables. As the size of the plaque varies in intervals of 1 or $2 \mathrm{~mm}$ from $11 \mathrm{~mm}$ to $22 \mathrm{~mm}$, we considered this variable as continuous. Patient characteristics were summarized as mean, standard deviation (SD), median, and interquartile range (IQR) for continuous variables and proportions for categorical variables.

All analyses were conducted using SPSS version 24.0 (IBM, Somers, NY, USA) and XLSTAT version 2016.02.28451 (Addinsoft). Statistical significance level was set at 0.05 .

\section{Results}

\section{Patients}

From 1996 through June 2016, 243 cases of choroidal melanoma were treated with ophthalmic brachytherapy. Initial level of VA in VAS scale more than $50(\mathrm{~V}>0.1)$ was noted in 185 patients $(76.1 \%)$ fulfilling the inclusion criteria. A total of 1,668 examinations were performed, with a median follow-up of 74.2 months (range, 3-223) with loss of follow-up of less than $1 \%$.

VA score in V and VAS scales were available in all patients. VA on presentation was $20 / 12.5$ to $20 / 20$ in 54 patients $(29 \%), 20 / 20$ to $20 / 32$ in 44 patients $(24 \%)$, $20 / 32$ to $20 / 50$ in 44 patients (24\%), 20/50 to $20 / 80$ in 21 patients (11\%), $20 / 80$ to $20 / 200$ in 22 patients $(12 \%)$, and $20 / 200$ to $20 / 125$ in 1 patients $(1 \%)$. Corresponding to the intervals of equal width 100-110, 90-100, 80-90, 70-80, 60-70, and 50-60 in VAS scale, respectively.

Baseline patient demographic and tumor characteristics are summarized in Tables 1 and 2. Physical doses and BED to tumor apex, sclera, optic disc, foveola, and lens are presented in Table 3.

\section{Temporal analysis and model fitting}

Data representing the two variables $(\Delta(T)$ vs. $T)$ are displayed in a two-dimensional scatter plot. Best fit corresponds to the exponential model with the following functional form: $\Delta(T)=\mathrm{ae}^{-\mathrm{bT}}$ (Figure 1), with a correla- 
Table 1. Tumor and treatment features. Quantitative variables, $N=185$

\begin{tabular}{lcccccc} 
Variable & Minimum & Maximum & Mean & SD & Median & IQR \\
\hline Age (years) & 16.00 & 87.00 & 59.46 & 13.16 & 61.00 & $51.00-69.00$ \\
\hline Tumor apical height $(\mathrm{mm})$ & 1.00 & 12.11 & 5.49 & 2.39 & 5.13 & $3.53-7.25$ \\
\hline Longest basal dimension $(\mathrm{mm})$ & 5.00 & 20.47 & 11.39 & 2.75 & 11.50 & $9.27-13.46$ \\
\hline Total source strength $(\mathrm{U})$ & 13.56 & 128.14 & 58.83 & 24.02 & 55.44 & $40.55-76.00$ \\
\hline Treatment time $(\mathrm{h})$ & 58.00 & 283.00 & 128.21 & 43.48 & 103.00 & $95.00-167.00$ \\
\hline Size of the plaque $(\mathrm{mm})$ & 11.00 & 22.00 & 16.00 & 2.24 & 16.00 & $14.00-18.00$
\end{tabular}

$S D$ - standard deviation, $I Q R$ - interquartile range

tion of $R^{2}=0.81$. Fit values and standard errors (SE) are: $\mathrm{a}=83.97(\mathrm{SE}, 4.56), \mathrm{b}=0.22(\mathrm{SE}, 0.03) . R^{2}$ values for linear, quadratic, and hyperbolic fits were $0.80,0.78$, and 0.71 , respectively.

Table 2. Tumor and treatment features. Qualitative variables, $N=185$

\begin{tabular}{|c|c|c|c|}
\hline $\begin{array}{l}\text { Variable/ } \\
\text { statistic }\end{array}$ & Categories & $n$ & $\%$ \\
\hline \multirow[t]{3}{*}{ COMS } & Large & 13 & 7.03 \\
\hline & Medium & 171 & 92.43 \\
\hline & Small & 1 & 0.54 \\
\hline \multirow[t]{2}{*}{ Gender } & Female & 104 & 56.22 \\
\hline & Male & 81 & 43.78 \\
\hline \multirow[t]{2}{*}{ Laterality } & Right eye & 97 & 52.43 \\
\hline & Left eye & 88 & 47.57 \\
\hline \multirow[t]{2}{*}{ Length } & Nasal & 50 & 27.03 \\
\hline & Temporal & 135 & 72.97 \\
\hline \multirow[t]{2}{*}{ Latitude } & Inferior & 75 & 40.54 \\
\hline & Superior & 110 & 59.46 \\
\hline \multirow{3}{*}{$\begin{array}{l}\text { Location of } \\
\text { anterior tumor } \\
\text { border }\end{array}$} & Ciliary body & 19 & 10.27 \\
\hline & Equator to ora serrata & 74 & 40.00 \\
\hline & Posterior to equator & 92 & 49.73 \\
\hline \multirow{4}{*}{$\begin{array}{l}\text { Location of } \\
\text { posterior } \\
\text { border }\end{array}$} & $<1 \mathrm{~mm}$ OD & 19 & 10.27 \\
\hline & $>1 \mathrm{~mm}$ OD & 157 & 84.86 \\
\hline & Ciliary body & 1 & 0.54 \\
\hline & Equator to ora serrata & 8 & 4.32 \\
\hline \multirow[t]{3}{*}{ Tumor shape } & Mushroom. & 33 & 17.84 \\
\hline & Diffuse & 2 & 1.08 \\
\hline & Nodular & 150 & 81.08 \\
\hline \multirow{2}{*}{$\begin{array}{l}\text { Juxtapapillary } \\
\text { localization* }\end{array}$} & No & 158 & 85.41 \\
\hline & Yes & 27 & 14.59 \\
\hline \multirow[t]{2}{*}{ Shape plaque } & Notched & 26 & 14.05 \\
\hline & Not notched & 159 & 85.95 \\
\hline \multirow[t]{2}{*}{ Type of plaque } & COMS & 145 & 78.38 \\
\hline & ROPES & 40 & 21.62 \\
\hline
\end{tabular}

*uxtapapillary choroidal melanoma is considered with a posterior margin with in $1 \mathrm{~mm}$ of the optic disc (OD)

\section{Kaplan-Meier analysis}

At last follow-up, 91/185 (49\%) patients recorded visual acuity better than $20 / 200(\mathrm{~V}>0.1)$. A decline in VAS to 50 ( 0.1 in V scale) or worse was observed in 94 patients, with a survival mean time of 39 months (95\% CI: 32.0457.96). Actuarial Kaplan-Meier curves and confidence intervals are described in Figure 2. VA preservation rates at 3, 5, 10, and 15 years were $57 \%$ (95\% CI: 48-66\%), $42 \%$ (95\% CI: $33-51 \%$ ), $27 \%$ (95\% CI: $16-37 \%$ ), and $23 \%$ (95\% CI: 12-34\%), respectively.

\section{Cox analysis}

Univariate factors predictive of poor VA are listed in Table 4 showing that, among others features, many doses and BEDs' to the different tissues are statistically significant. Multivariate logistic regression analysis, including age, revealed (Table 5) that tumor apical height $(\mathrm{HR}=1.18$, 95\% CI: 1.07-1.29, $p<0.001)$, plaque size $(\mathrm{HR}=1.22$, 95\% CI: 1.08-1.36, $p<0.002)$, juxtapapillary localization $(\mathrm{HR}=1.70,95 \% \mathrm{CI}: 1.01-2.84, p=0.044)$, and dose to foveola (HR $=1.01,95 \%$ CI: 1.00-1.01, $p=0.005)$ were significant. BED's dependencies vanished when multivariate competing risks regression modeling was performed.

\section{Log-rank test}

Figure 3 shows the study of survival curves for the relevant variables of the multivariate study, where the curves are separated according to their median value for the quantitative variables and according to their category in the qualitative ones. All four variables (apical height, plaque size, juxtapapillary localization, and dose to foveola) analyzed by the log-rank test reported $p$ values less than 0.05 , so the survival curves differ significantly.

\section{Discussion}

In this report, we present our experience of VA outcome after plaque brachytherapy and the possible relationship with clinical features including radiobiological doses, among others, in a large series of patients from a single center. To our knowledge, our study is the first to model (by a mathematical function) VA deterioration after the treatment and to publish single-institutional follow-up data, which has been re-analyzed in attempt to convert physical doses to BED, and to correlate BED with late sequelae. Although there are many studies that 
Table 3. Dosimetric characteristics of plaque treatments of the cohort, $N=185$

\begin{tabular}{lcccccc} 
Variable & Minimum & Maximum & Mean & SD & Median & IQR \\
\hline Dose to tumor apex (Gy) & 74.65 & 94.86 & 85.24 & 3.40 & 85.15 & $84.00-86.76$ \\
\hline BED to tumor apex (Gy) & 87.36 & 138.26 & 108.87 & 9.85 & 109.65 & $100.70-116.04$ \\
\hline Dose to ON (Gy) & 2.93 & 136.85 & 33.13 & 22.35 & 28.54 & $16.57-42.64$ \\
\hline BED to ON (Gy) & 3.05 & 413.62 & 63.39 & 60.28 & 46.46 & $23.59-79.05$ \\
\hline Dose to lens (Gy) & 2.90 & 203.60 & 20.05 & 19.01 & 16.25 & $9.17-26.15$ \\
\hline BED to lens (Gy) & 3.21 & $1,118.71$ & 38.47 & 85.45 & 22.86 & $12.08-41.33$ \\
\hline Dose to foveola (Gy) & 3.17 & 268.00 & 43.76 & 37.22 & 33.70 & $18.68-53.94$ \\
\hline BED to foveola (Gy) & 3.27 & 987.96 & 91.52 & 127.56 & 48.52 & $23.93-94.46$ \\
\hline Dose to sclera (Gy) & 82.34 & 722.00 & 284.72 & 125.30 & 252.20 & $202.30-348.15$ \\
\hline BED to sclera (Gy) & 140.10 & $4,575.32$ & $1,313.23$ & 861.85 & $1,059.12$ & $740.60-1,674.93$ \\
\hline Dose to EC (Gy) & 8.49 & 85.62 & 30.07 & 14.66 & 26.24 & $18.92-37.96$
\end{tabular}

$B E D$ - biologically effective dose, SD - standard deviation, IQR - interquartile range

attempt to model VA deterioration such as by Shields et al. [39], none of them has established a mathematical function that evaluates the progressive loss over time. A multi-center study involving larger patient population may confirm or disprove the findings.

Although the preservation of the organ is achieved in the majority of patients (95\%), a significative number of them experienced a decrease in VA secondary to therapy. We should mention that brachytherapy could be used only for designated tumors, depending mainly on their size and location.

By the method of quarterly averages, we smooth out random fluctuations of data and generate a forecast value for the period of analysis. $\Delta(\mathrm{T})$ are easy to calculate and, once plotted on a chart, is a powerful visual trend-spotting tool. On average, there is a worsening of the VA as time passes from the treatment. Five-year estimates (close to the mean time of the study), present a good adjustment coefficient, and shows a time reduction by $25 \%$ in VAS score of 5 months and a time reduction by $50 \%$ of 27.8 months. About only a quarter of patients keeps a VAS > 50 in the affected organ at the end of this study by Kaplan-Meier estimations. For patients with a good initial VA, time remaining with VAS $>50$ is higher on average than those patients with a lower initial VA.

In agreement with other studies explaining the maintenance and progression of visual function, patients with a VAS $\leq 50(\mathrm{~V} \leq 0.1)$ on examination performed prior to brachytherapy were not included in the analysis. This represents a significant limitation in the study because some of these patients gain VA as a result of the treatment, improving the mark of the initial exploration. Another important bias occurs when loss of VA after treatment results from a cataract (radiation induced or not). The surgery may help regain an important part of the VA lost but, due to the survival analysis performed, once an event has happened, the subject left the study and did not re-enter. On the other hand, VA measurement can be challenging because there are no specific standards related to the type of use to which the test is designed [40]. This stresses the difficulties in comparing studies from different institutions of the patient populations. In spite of it,

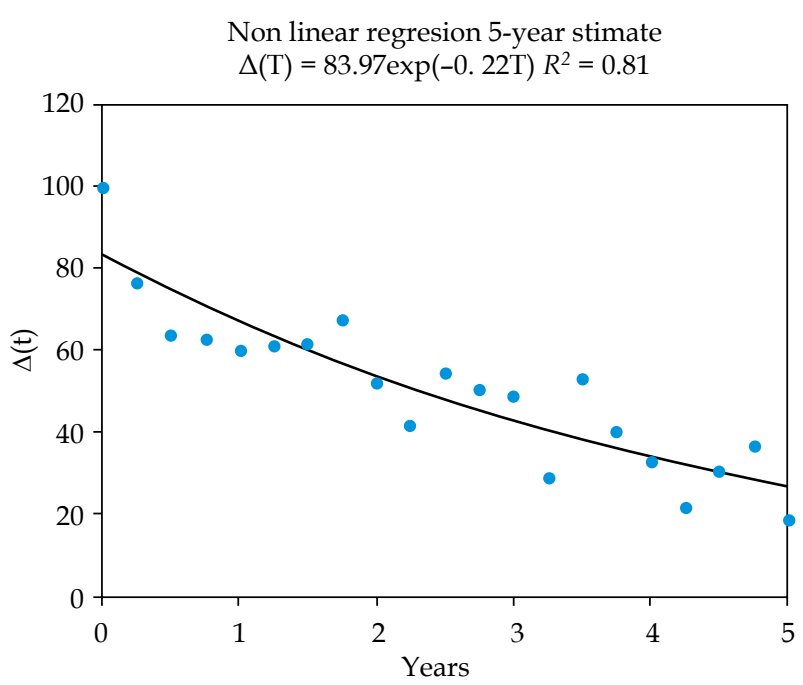

Fig. 1. Scatterplot and fitted regression line. Presentation of the mean $\Delta(\mathrm{T})$ and quarter, $\mathrm{T}$, after brachytherapy for 5 years

patients in our series experienced visual outcomes comparable to those in other reported series $[9,41,42,43,44]$.

The results of the present study show the association between visual loss and BED to tumor and critical tissues, and was statistically significant, but not on multivariate analysis. Important variables in multivariate model are the apical height, the size of the plaque, juxtapapillary localization, and the dose to the foveola. The first two are linked to tumor size. The larger it will be, the more and critical changes in ocular structures will occur. On the other hand, linked with the size of the tumor, the amount of radiation received by the whole eye will generally be greater. Juxtapapillary localization is linked largely to cases of invasion to the optic nerve and therefore is inexorably associated with higher doses to this tissue. The foveola, by proximity, will receive more doses, on average.

Multivariate studies carried out by some authors, obtained significant values in many different features depending on the variables analyzed: greater size $[41,42,43,45,46,47,48,49]$, smaller distance to the fovea or macula $[9,41,43,45,46,47,48,50,51]$, smaller distance to the 
Cumulative survival funtion. VA preservation

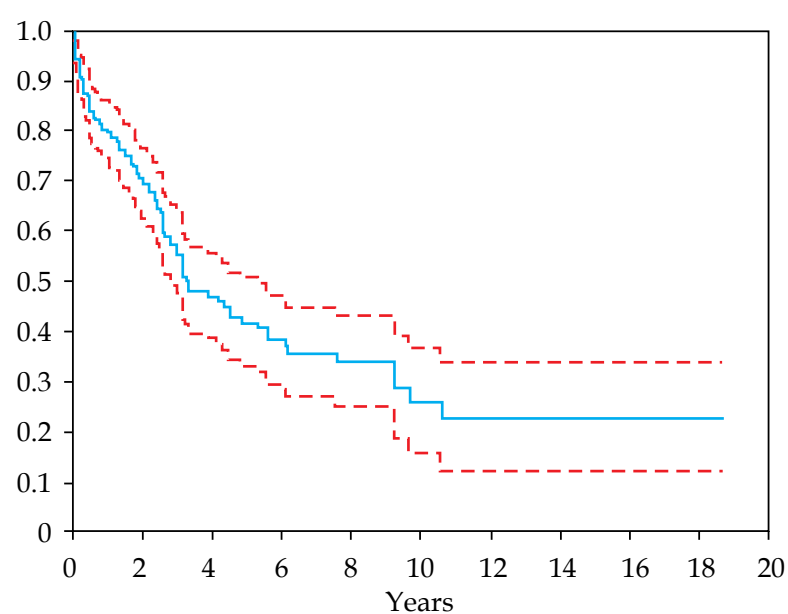

\begin{tabular}{|l|c|c|c|c|c|c|c|c|c|c|c|c|c|c|c|c|c|c|c|}
\hline T(yr) & $\mathbf{0}$ & $\mathbf{1}$ & $\mathbf{2}$ & $\mathbf{3}$ & $\mathbf{4}$ & $\mathbf{5}$ & $\mathbf{6}$ & $\mathbf{7}$ & $\mathbf{8}$ & $\mathbf{9}$ & $\mathbf{1 0}$ & $\mathbf{1 1}$ & $\mathbf{1 2}$ & $\mathbf{1 3}$ & $\mathbf{1 4}$ & $\mathbf{1 5}$ & $\mathbf{1 6}$ & $\mathbf{1 7}$ & $\mathbf{1 8}$ \\
\hline $\mathbf{N}$ & 185 & 136 & 99 & 65 & 47 & 38 & 31 & 23 & 19 & 15 & 10 & 6 & 6 & 5 & 5 & 5 & 3 & 3 & 1 \\
\hline SP & 100 & 80 & 70 & 57 & 47 & 42 & 38 & 35 & 34 & 34 & 27 & 27 & 23 & 23 & 23 & 23 & 23 & 23 & 23 \\
\hline
\end{tabular}

Fig. 2. Actuarial Kaplan-Meier, curve in blue, for the event VAS $\leq 50$. Values at $95 \%$ CI are in red. Mortality Table is below. $N$ is the number of patients at risk and SP is the survival probability (\%)

Table 4. Univariable Cox regression analysis of visual lost. Factors associated with follow-up VAS of 50 or worse

\begin{tabular}{|c|c|c|c|c|}
\hline \multirow[t]{2}{*}{ Variable } & \multirow[t]{2}{*}{$\mathrm{HR}$} & \multicolumn{2}{|c|}{$95 \% \mathrm{Cl}$ for $\mathrm{HR}$} & \multirow[t]{2}{*}{$p$ value } \\
\hline & & Lower & Top & \\
\hline Tumor apical height (mm) & 1.263 & 1.157 & 1.377 & 0.000 \\
\hline Longest basal dimension (mm) & 1.144 & 1.060 & 1.234 & 0.001 \\
\hline Activity (U) & 1.023 & 1.015 & 1.032 & 0.000 \\
\hline Plaque size $(\mathrm{mm})$ & 1.301 & 1.180 & 1.434 & 0.000 \\
\hline Dose OD (Gy) & 1.016 & 1.009 & 1.024 & 0.000 \\
\hline BED OD (Gy) & 1.005 & 1.002 & 1.008 & 0.000 \\
\hline Dose to lens (Gy) & 1.015 & 1.007 & 1.023 & 0.000 \\
\hline BED to lens (Gy) & 1.003 & 1.001 & 1.004 & 0.001 \\
\hline Dose to foveola (Gy) & 1.007 & 1.004 & 1.011 & 0.000 \\
\hline BED to foveola (Gy) & 1.001 & 1.000 & 1.002 & 0.005 \\
\hline Dose to sclera (Gy) & 1.004 & 1.002 & 1.005 & 0.000 \\
\hline BED sclera (Gy) & 1.001 & 1.000 & 1.001 & 0.000 \\
\hline Dose to EC (Gy) & 1.035 & 1.022 & 1.048 & 0.000 \\
\hline \multicolumn{5}{|l|}{ Tumor shape } \\
\hline Mushroom (Ref) & & & & 0.010 \\
\hline Diffuse & 0.898 & 0.212 & 3.804 & 0.884 \\
\hline Nodular & 0.501 & 0.317 & 0.790 & 0.003 \\
\hline Juxtapapillary localization (yes vs. no) & 1.687 & 1.018 & 2.796 & 0.042 \\
\hline
\end{tabular}

$B E D$ - biologically effective dose, HR - hazard ratio, EC - eye center 
Table 5. Multivariable Cox regression analysis of visual lost. Factors associated with follow-up VAS $\leq 50$

\begin{tabular}{llccc} 
Factor & HR & \multicolumn{2}{c}{$95 \% \mathrm{Cl}$ for HR } & \multirow{2}{*}{$p$ value } \\
\cline { 3 - 4 } & & Lower & Top & $<0.001$ \\
\hline Tumor apical height (mm) & 1.177 & 1.071 & 1.293 & $<0.001$ \\
\hline Plaque size (mm) & 1.215 & 1.084 & 1.362 & 0.044 \\
\hline Juxtapapillary localization (yes vs. no) & 1.698 & 1.014 & 2.844 & 0.005
\end{tabular}

$H R$ - hazard ratio

A

A

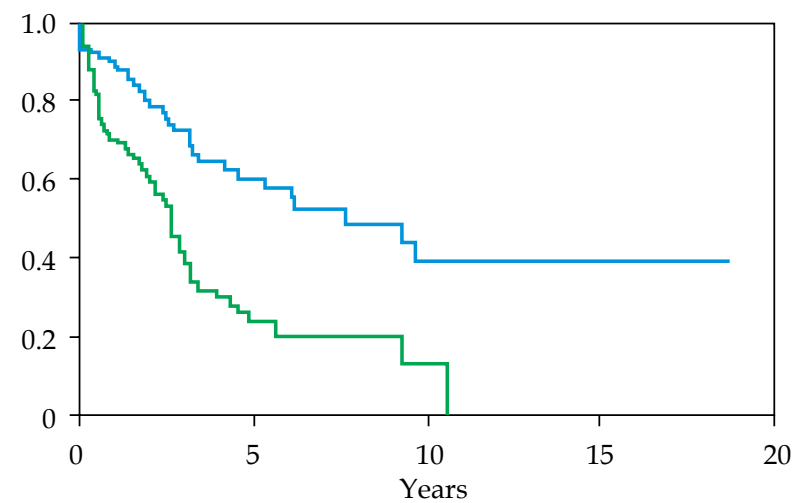

$\longrightarrow 5.13 \mathrm{~mm} \stackrel{\text { 工 }}{\longrightarrow} 5.13 \mathrm{~mm}$

\begin{tabular}{|c|l|c|c|c|c|c|c|c|}
\hline & $\mathbf{T}(\mathbf{y r})$ & $\mathbf{0}$ & $\mathbf{1}$ & $\mathbf{3}$ & $\mathbf{5}$ & $\mathbf{1 0}$ & $\mathbf{1 5}$ & $\mathbf{1 8}$ \\
\hline$\leq 5.13 \mathrm{~mm}$ & $\mathrm{~N}$ & 93 & 78 & 40 & 27 & 8 & 5 & 1 \\
\cline { 2 - 9 } & $\mathrm{SP}$ & 100 & 89 & 72 & 60 & 39 & 39 & 39 \\
\hline$>5.13 \mathrm{~mm}$ & $\mathrm{~N}$ & 92 & 58 & 25 & 12 & 2 & - & - \\
\cline { 2 - 9 } & $\mathrm{SP}$ & 100 & 69 & 39 & 24 & 13 & - & - \\
\hline
\end{tabular}

C

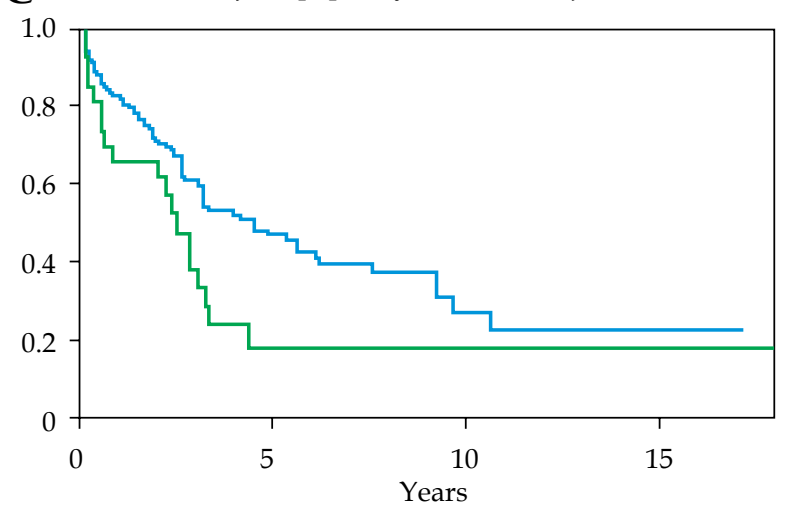

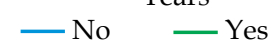

\begin{tabular}{|l|l|c|c|c|c|c|c|c|}
\hline & $\mathbf{T}(\mathbf{y r})$ & $\mathbf{0}$ & $\mathbf{1}$ & $\mathbf{3}$ & $\mathbf{5}$ & $\mathbf{1 0}$ & $\mathbf{1 5}$ & $\mathbf{1 8}$ \\
\hline \multirow{2}{*}{ No } & $\mathrm{N}$ & 158 & 119 & 57 & 34 & 8 & 4 & - \\
\cline { 2 - 10 } & $\mathrm{SP}$ & 100 & 82 & 60 & 47 & 27 & 23 & - \\
\hline \multirow{3}{*}{ Yes } & $\mathrm{N}$ & 27 & 17 & 8 & 3 & 2 & 1 & 1 \\
\cline { 2 - 9 } & $\mathrm{SP}$ & 100 & 66 & 33 & 18 & 18 & 18 & 18 \\
\hline
\end{tabular}

B CSF plaque size, $p<0.0001$

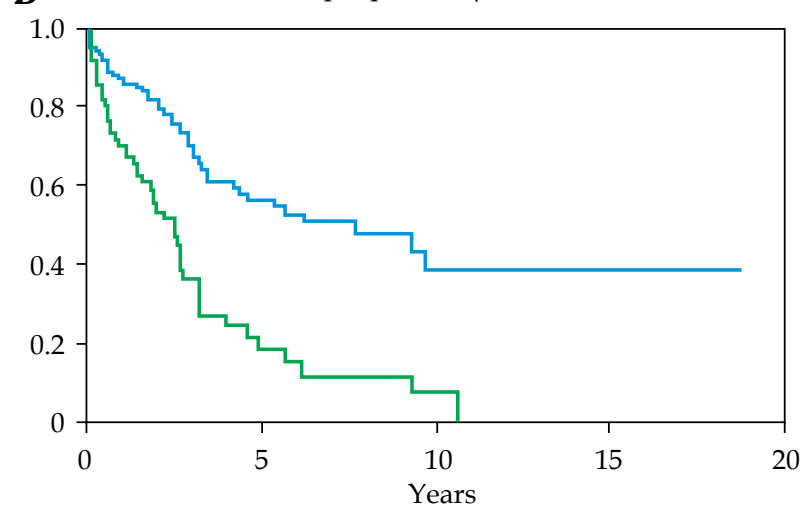

$\longrightarrow \leq 16 \mathrm{~mm} \quad \longrightarrow 16 \mathrm{~mm}$

\begin{tabular}{|l|l|c|c|c|c|c|c|c|}
\hline & $\mathbf{T}(\mathbf{y r})$ & $\mathbf{0}$ & $\mathbf{1}$ & $\mathbf{3}$ & $\mathbf{5}$ & $\mathbf{1 0}$ & $\mathbf{1 5}$ & $\mathbf{1 8}$ \\
\hline$\leq 16 \mathrm{~mm}$ & $\mathrm{~N}$ & 109 & 91 & 49 & 32 & 7 & 5 & 1 \\
\cline { 2 - 10 } & $\mathrm{SP}$ & 100 & 86 & 67 & 56 & 38 & 38 & 38 \\
\hline$>16 \mathrm{~mm}$ & $\mathrm{~N}$ & 76 & 45 & 16 & 6 & 2 & - & - \\
\cline { 2 - 9 } & $\mathrm{SP}$ & 100 & 67 & 27 & 15 & 7 & - & - \\
\hline
\end{tabular}

D Dose to foveola, $p<0.0001$

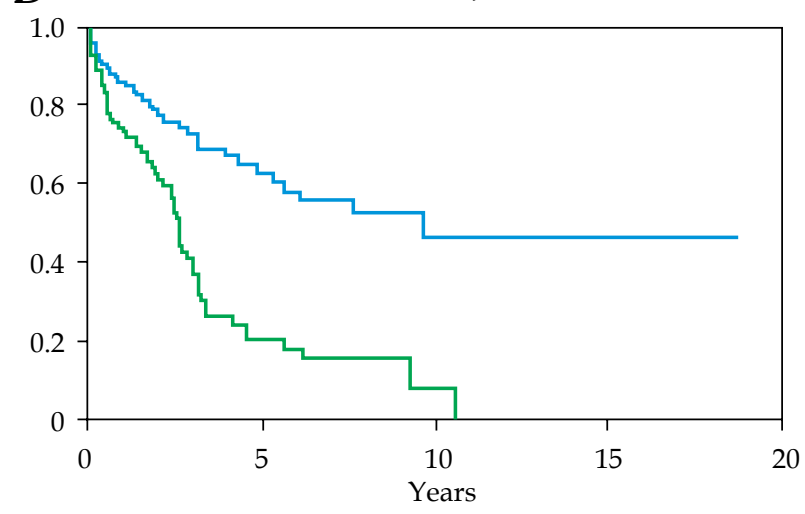

$\longrightarrow 33.70$ Gy $\quad>33.70 \mathrm{~Gy}$

\begin{tabular}{|c|l|c|c|c|c|c|c|c|}
\hline & $\mathbf{T}(\mathbf{y r})$ & $\mathbf{0}$ & $\mathbf{1}$ & $\mathbf{3}$ & $\mathbf{5}$ & $\mathbf{1 0}$ & $\mathbf{1 5}$ & $\mathbf{1 8}$ \\
\hline \multirow{3}{*}{$\leq 33.70$ Gy } & $\mathrm{N}$ & 93 & 72 & 42 & 28 & 7 & 5 & 1 \\
\cline { 2 - 10 } & $\mathrm{SP}$ & 100 & 85 & 73 & 63 & 46 & 46 & 46 \\
\hline \multirow{3}{*}{$>33.70$ Gy } & $\mathrm{N}$ & 92 & 61 & 23 & 9 & 2 & - & - \\
\cline { 2 - 9 } & $\mathrm{SP}$ & 100 & 73 & 37 & 18 & 5 & - & - \\
\hline
\end{tabular}

Fig. 3. Kaplan-Meier cumulative survival functions (CSF) for the event VAS $\leq 50$. The curves in each of the graphs are not overlapping and there is statistically significant difference between the for the variables tumor height, plaque size, juxtapapillary localization and dose to fovea by log-rank test $(p<0.05)$. For survival tables $N$ is the number of patients in the time interval and $\mathrm{SP}$ is the survival probability in \% 
optic nerve $[9,47,50,52,53]$, dose to optic nerve [42,45], dose to sclera [42], dose to macula [42], high macula dose rates [54], older age [43], younger age [45], initial visual acuity [45], retinal invasion prior to treatment [52], tumor shape $[46,53]$, plaque shape [53], diabetes mellitus [46,49], and serous macular detachment [46]. Finally, compared with doses of prescription of $85 \mathrm{~Gy}$, some previous studies had shown that lower doses of radiation had correlated with lower rates of visual loss $[55,56]$.

When separately analyzing survival curves from variables with statistical significance in the multivariate study, patients with a tumor height greater than $5.13 \mathrm{~mm}$, plaque size greater than $16 \mathrm{~mm}$, dose to foveola higher than 33.7 Gy, and juxtapapillary location had significative worse prognostic. We should be careful in interpreting this results for several seasons. Firstly, the decision to bifurcate the VA analysis of these variables about these values is a function of the cohort characteristics. Secondly, Kaplan-Meier curves behave poorly in the tails and the reliability of estimates is intuitively bad when there are less than $10 \%$ patients remaining in the cohort [57].

The results of this study could help us to preserve patients' VA when planning therapies, and the total dose to foveola and the plaque size being the only relevant adjustable parameters. Other relevant, but not controllable features, were juxtapapillary localization and apical height at diagnosis.

These conclusions were not clearly supported by our radiobiological studies, since VA deterioration is a result of a tissue damage. Within the time range of conventional lowdose-rate for episcleral brachytherapy $(0.6$ to $1.05 \mathrm{~Gy} / \mathrm{h})$ and for tissues with low $\mathrm{\alpha} / \beta$ ratio repair, kinetics plays not significant role [58]. The opposite situation is supposed for tumor tissue with a $\alpha / \beta$ ratio of 10 Gy or more [58]. Therefore, it is assumed to keep the total dose high to maximize local control, and BED to OAR low to minimize late toxicities.

The main limitations of the radiobiological modeling are the lack of control of all the biological mechanisms involved in the expression of a certain radiation effect, and the uncertainties in the formulation of the models from clinical data [59]. Therefore, these models may not reflect normal tissue complications properly and as a result, the analysis could fail. This may be why the correlations between BED and VA loss has not been very consistent to date. It could be that lower doses as reported recently in the literature $[55,56,60]$, should be sufficient to achieve acceptable local control rates in patients, and radiobiological dose could have an important role when analyzing VA loss.

\section{Conclusions}

VA changes can be modeled by a negative exponential function of two parameters with a strong correlation for the first 5 years after treatment. The reported results show a relationship with the physical dose to the foveola and visual outcome in patients after brachytherapy. This relationship is not clear for all the radiobiological doses analyzed that are statistically significant in the univariate study, but not in the multivariable one. Other risk factors for poor visual outcome were apical height, size of the plaque, and juxtapapillary localization.

\section{Disclosure}

The authors report no conflict of interest.

D. Miguel's contributions form part of his PhD thesis under the supervision of F. López-Lara and J. de FrutosBaraja at the University of Valladolid.

\section{References}

1. Singh AD, Turell ME, Topham AK. Uveal melanoma: trends in incidence, treatment, and survival. Ophthalmology 2011; 118: 1881-1885.

2. Margo CE. The Collaborative Ocular Melanoma Study: an overview. Cancer Control 2004; 11: 304-309.

3. Rao YJ, Sein J, Badiyan S et al. Patterns of care and survival outcomes after treatment for uveal melanoma in the postcoms era (2004-2013): a surveillance, epidemiology, and end results analysis. J Contemp Brachytherapy 2017; 9: 453-465.

4. Singh AD, Kivelä T. The collaborative ocular melanoma study. Ophthalmol Clin North Am 2005; 18: 129-142.

5. ICRU. Dosimetry of Beta rays and low-energy photons for brachytherapy with sealed sources. J ICRU 2004; 4: 21-89.

6. Nag S, Quivey JM, Earle JD et al. The American Brachytherapy Society recommendations for brachytherapy of uveal melanomas. Int J Radiat Oncol Biol Phys 2003; 56: 544-555.

7. Damato B. Progress in the management of patients with uveal melanoma. The 2012 Ashton Lecture. Eye 2012; 26: 1157-1172.

8. Simpson ER, Gallie B, Laperrierre N et al. The American Brachytherapy Society consensus guidelines for plaque brachytherapy of uveal melanoma and retinoblastoma. Brachytherapy 2014; 13: 1-14

9. Jensen AW, Petersen IA, Kline RW et al. Radiation complications and tumor control after 125I plaque brachytherapy for ocular melanoma. Int J Radiat Oncol Biol Phys 2005; 63: 101-108.

10. Brady LW, Shields J, Augusburger J et al. Complications from radiation therapy to the eye. Front Radiat Ther Oncol 1989; 23: 238-254.

11. Gündüz K, Shields CL, Shields JA et al. Radiation retinopathy following plaque radiotherapy for posterior uveal melanoma. Arch Ophthalmol 1999; 117: 609-614.

12. Fontanesi J, Meyer D, Xu S et al. Treatment of choroidal melanoma with I-125 plaque. Int J Radiat Oncol Biol Phys 1993; 26: 619-623.

13. Lommatzsch PK. Results after beta-irradiation (106Ru/106Rh) of choroidal melanomas. Twenty years' experience. Am J Clin Oncol 1987; 10: 146-151.

14. Summanen $\mathrm{P}$, Immonen $\mathrm{I}$, Kivelä $\mathrm{T}$ et al. Radiation related complications after ruthenium plaque radiotherapy of uveal melanoma. Br J Ophthalmol 1996; 80: 732-739.

15. Groenewald C, Konstantinidis L, Damato B. Effects of radiotherapy on uveal melanomas and adjacent tissues. Eye (Lond) 2013; 27: 163-171.

16. Tagliaferri L, Pagliara MM, Masciocchi C et al. Nomogram for predicting radiation maculopathy in patients treated with Ruthenium-106 plaque brachytherapy for uveal melanoma. J Contemp Brachytherapy 2017; 9: 540-547.

17. Rospond-Kubiak I, Wróblewska-Zierhoffer M, TwardoszPawlik $\mathrm{H}$ et al. Ruthenium brachytherapy for uveal melanoma - single institution experience. J Contemp Brachytherapy 2017; 9: 548-552.

18. Parsons JT, Bova FJ, Mendenhall WM et al. Response of the normal eye to high dose radiotherapy. Oncology (Williston Park) 1996; 10: 837-847.

19. Dale RG. Some theoretical derivations relating to the tissue dosimetry of brachytherapy nuclides.pdf. Med Phys 1983; 10: 176-183. 
20. Gagne NL, Leonard KL, Huber KE et al. BEDVH-A method for evaluating biologically effective dose volume histograms: application to eye plaque brachytherapy implants. Med Phys 2012; 39: 976-983.

21. Gagne NL, Leonard KL, Rivard MJ. Radiobiology for eye plaque brachytherapy and evaluation of implant duration and radionuclide choice using an objective function. Med Phys 2012; 39: 3332-3342.

22. Tagliaferri L, Pagliara MM, Boldrini L et al. INTERACTS (INTErventional Radiotherapy ACtive Teaching School) guidelines for quality assurance in choroidal melanoma interventional radiotherapy (brachytherapy) procedures. J Contemp Brachytherapy 2017; 9: 287-295.

23. Granero D, Pérez-Calatayud J, Ballester F et al. Dosimetric study of the $15 \mathrm{~mm}$ ROPES eye plaque. Med Phys 2004; 31: 3330-3336.

24. Chiu-Tsao S-T, Astrahan MA, Finger PT et al. Dosimetry of (125)I and (103)Pd COMS eye plaques for intraocular tumors: report of Task Group 129 by the AAPM and ABS. Med Phys 2012; 39: 6161-6184.

25. Bailey IL, Lovie-Kitchin JE. Visual acuity testing. From the laboratory to the clinic. Vision Res 2013; 90: 2-9.

26. Masin SC, Zudini V, Antonelli M. Early alternative derivations of Fechner's law. J Hist Behav Sci 2009; 45: 56-65.

27. Gagne NL, Rivard MJ. Quantifying the dosimetric influences of radiation coverage and brachytherapy implant placement uncertainty on eye plaque size selection. Brachytherapy 2013; 12: 508-520.

28. Astrahan MA, Luxton G, Jozsef G et al. An interactive treatment planning system for ophthalmic plaque radiotherapy. Int J Radiat Oncol Biol Phys 1990; 18: 679-687.

29. Sloboda RS, Menon GV. Experimental determination of the anisotropy function and anisotropy factor for model 6711 I-125 seeds. Med Phys 2000; 27: 1789-1799.

30. Plagnard J, Oliveira C, Cutarella D et al. Full characterization of the 125 I IBt Bebig I25.S16 brachytherapy source and sensitivity study of the absorbed dose to water due to the seed dimensional variations. Metrologia 2012; 49: 223-227.

31. Nath R, Anderson LL, Luxton G et al. Dosimetry of interstitial brachytherapy sources: recommendations of the AAPM Radiation Therapy Committee Task Group No. 43. American Association of Physicists in Medicine. Med Phys 1995; 22: 209-234.

32. Rivard MJ, Coursey BM, DeWerd LA et al. Update of AAPM Task Group No. 43 Report: A revised AAPM protocol for brachytherapy dose calculations. Med Phys 2004; 31: 633674.

33. Gagne NL, Cutright DR, Rivard MJ. Keeping an eye on the ring: COMS plaque loading optimization for improved dose conformity and homogeneity. J Contemp Brachytherapy 2012; 4: $165-175$.

34. Dale RG, Jones B. The clinical radiobiology of brachytherapy. Br J Radiol 1998; 71: 465-483.

35. Baltas D, Lymperopoulou G, Löffler E et al. A radiobiological investigation on dose and dose rate for permanent implant brachytherapy of breast using 125I or 103Pd sources. Med Phys 2010; 37: 2572-2586.

36. Roberts SA, Hendry JH, Swindell R et al. Compensation for changes in dose-rate in radical low-dose-rate brachytherapy : a radiobiological analysis of a randomised clinical trial. $R a-$ diother Oncol 2004; 70: 63-74.

37. Bland JM, Altman DG. Survival probabilities (the Kaplan-Meier method). BMJ 1998; 317: 1572.

38. Lunn M, McNeil D. Applying Cox regression to competing risks. Biometrics 1995; 51: 524-532.

39. Shields CL, Shields JA, Cater J et al. Plaque radiotherapy for uveal melanoma: long-term visual outcome in 1106 consecutive patients. Arch Ophthalmol 2000; 118: 1219-1228.
40. Ferris FL 3rd, Bailey I. Standardizing the measurement of visual acuity for clinical research studies: Guidelines from the Eye Care Technology Forum. Ophthalmology 1996; 103: 181-182.

41. Melia BM, Abramson DH, Albert DM et al. Collaborative ocular melanoma study (COMS) randomized trial of I-125 brachytherapy for medium choroidal melanoma. I. Visual acuity after 3 years COMS report no. 16. Ophthalmology 2001; 108: 348-366.

42. Wagner A, Chen A, Cook T et al. Outcomes and Control Rates for I-125 Plaque Brachytherapy for Uveal Melanoma: A Community-Based Institutional Experience. ISRN Ophthalmol 2014; 2014: 950975.

43. Khan N, Khan MK, Bena J et al. Plaque brachytherapy for uveal melanoma: a vision prognostication model. Int J Radiat Oncol Biol Phys 2012; 84.

44. Damato B, Patel I, Campbell IR et al. Visual acuity after Ruthenium106 brachytherapy of choroidal melanomas. Int J Radiat Oncol Biol Phys 2005; 63: 392-400.

45. Aziz HA, Singh N, Bena J et al. Vision Loss Following Episcleral Brachytherapy for Uveal Melanoma. JAMA Ophthalmol 2016; 134.

46. Lumbroso-Le Rouic L, Charif Chefchaouni M, Levy C et al. 125I plaque brachytherapy for anterior uveal melanomas. Eye (Lond) 2004; 18: 911-916.

47. Quivey JM, Augsburger J, Snelling L et al. 125I plaque therapy for uveal melanoma. Analysis of the impact of time and dose factors on local control. Cancer 1996; 77: 2356-2362.

48. Bosworth JL, Packer S, Rotman M et al. Choroidal melanoma: I-125 plaque therapy. Radiology 1988; 169: 249-251.

49. Packer S, Rotman M. Radiotherapy of choroidal melanoma with iodine-125. Ophthalmology 1980; 87: 582-590.

50. Garretson BR, Robertson DM, Earle JD. Choroidal melanoma treatment with iodine 125 brachytherapy. Arch Ophthalmol 1987; 105: 1394-1397.

51. Puusaari I, Heikkonen J, Kivelä T. Ocular complications after iodine brachytherapy for large uveal melanomas. Ophthalmology 2004; 111: 1768-1777.

52. Shields CL, Naseripour M, Cater J et al. Plaque Radiotherapy for Large Posterior Uveal Melanomas (> 8-mm thick) in 354 Consecutive Patients. Ophthalmology 2002; 109: 1838-1849.

53. Naseripour M, Jaberi R, Sedaghat A et al. Ruthenium-106 brachytherapy for thick uveal melanoma: reappraisal of apex and base dose radiation and dose rate. J Contemp Brachytherapy 2016; 8: 66-73.

54. Jones R, Gore E, Mieler W et al. Posttreatment visual acuity in patients treated with episcleral plaque therapy for choroidal melanomas: dose and dose rate effects. Int J Radiat Oncol Biol Phys 2002; 52: 989-995.

55. Murray TG, Markoe AM, Gold AS et al. Long-term follow up comparing two treatment dosing strategies of $125 \mathrm{I}$ plaque radiotherapy in the management of small/medium posterior uveal melanoma. J Ophthalmol 2013; 2013: 517032.

56. Perez BA, Mettu P, Vajzovic L et al. Uveal melanoma treated with iodine-125 episcleral plaque: An analysis of dose on disease control and visual outcomes. Int J Radiat Oncol Biol Phys 2014; 89: 127-136.

57. Rich JT, Neely JG, Paniello RC et al. A practical guide to understanding Kaplan-Meier curves. Otolaryngol Head Neck Surg 2010; 143: 331-336.

58. Dutreix J. Expression of the dose rate effect in clinical curietherapy. Radiother Oncol 1989; 15: 25-37.

59. Fowler JF. 21 years of Biologically Effective Dose. Br J Radiol 2010: 554-568.

60. Gorovets D, Gagne NL, Melhus CS. Dosimetric and radiobiologic comparison of (103)Pd COMS plaque brachytherapy and Gamma Knife radiosurgery for choroidal melanoma. Brachytherapy 2017; 16: 433-443. 